\title{
E-learning and Knowledge Management: Context Structuration
}

\author{
Marco Pedroni \\ CARID University of Ferrara, Italy
}

marco.pedroni@unife.it

\begin{abstract}
Management of the cognitive context-understood as the reconstruction of the fabric of concepts and relations, representation in the form of a concept map and management of interactive functions that are inherent to or can be situated within the context itself-is a significant exigency both within the learning environment, and in particular for e-learning, as well as within the corporate environment as part of knowledge management processes.

In order to take advantage of knowledge mapping and the functions connected with it, software tools capable of supporting and managing interaction in a network environment must be designed and created on the basis of guidelines aimed at pursuing not only the ergonomics, efficiency and efficacy of use, but also flexibility and completeness during the phases of construction, updating and analysis of the relational fabric.
\end{abstract}

Keywords: concept maps, knowledge management, e-learning, context structure, knowledge sharing.

\section{Introduction}

Among the learning strategies that underlie learning object structural models, generative learning has special importance given the specificity of the learning environment in which it may be applied: an environment comprised of highly-motivated students whose knowledge background is basically homogeneous and of a high level, and who are aware of the need to work together to find the solution to a problem and with the purpose of qualitatively improving the activity of the entire group, not just each individual student. This is the situation characteristic of those widespread and common situations in which, due to structural growth or re-engineering of procedures, there is a need for training within the workplace organization, training occurring between coworkers and not led by an instructor. Users involved in a learning process in which they must cooperate in identifying the problems to be tackled and resolved, the themes to be handled in a selfupdating process, the modes of learning interaction and action and, as a result, shared "metacognition", will configure their activity, in strategic terms, as generative learning.

Material published as part of this publication, either on-line or in print, is copyrighted by the Informing Science Institute. Permission to make digital or paper copy of part or all of these works for personal or classroom use is granted without fee provided that the copies are not made or distributed for profit or commercial advantage AND that copies 1) bear this notice in full and 2) give the full citation on the first page. It is permissible to abstract these works so long as credit is given. To copy in all other cases or to republish or to post on a server or to redistribute to lists requires specific permission and payment of a fee. Contact Publisher@InformingScience.org to request redistribution permission.

This strategy, when it expands to include learning tools based on the use of digital and online technologies (as a rule: learning is always among the top sectors that most rapidly assimilates innovations in communications technology), becomes so closely identified with knowledge management that the two processes cannot be distinguished from 
each other. In fact, if we take into consideration corporate knowledge management as an activity aimed at enhancing the knowledge and skills within the work environment through the presentation and sharing of tacit knowledge and the organization of shared or explicit knowledge (informational and documental patrimony), the overlap between this activity and generative learning is clear, both from a methodological standpoint and, consequently, from a technological one in the choice and implementation of suitable tools of interaction.

Starting from these premises, it is possible to design the technological features of a tool for online interaction configured to support generative learning and knowledge management and therefore with a two-fold possibility for fruition, and aimed both at sharing and contextualizing tacit and explicit knowledge to both guide metacognition and to analyze the context under examination in order to provide a decisional support tool.

\section{Context Management}

There are four axes (Pedroni, 2003) along which re-engineered learning activity runs, from a purely strategic standpoint (Frignani, 2003), through a design approach that takes into consideration technological innovation (learning activity in general, and not e-learning or blended learning organized as online training plus short attended seminars, because there is no question of the potential for applying the results of this re-engineering including in environments in which learning is primarily traditional in nature, i.e., normal, on-going classroom lessons). Added to the first two - content transmission and interaction between stakeholders and the training process, both of which are clear and therefore do not require further comment - are control of the training process (an axis which in online training takes the form of tracking activities, reports of the data collected and portfolio elaboration) and context management.

Here, we will concentrate on this last axis. By "context management" is meant the set of functions that permits the reconstruction of the knowledge context (a subject-based knowledge in formal and non-formal learning environments that becomes a patrimony of know-how, knowledge and skills within organizations and informal training environments) through graphic tools and on an individual and collaborative basis, its representation and use from a documental basis and as hermeneutic support, its availability on an ontological basis as a virtual site for negotiating meaning and metacognition, its use-value as a tool for navigation and progressive exploration of knowledge and, finally, its support role for the structured placement of documentary resources and asynchronous interaction around the topics examined.

From this definition, we can deduce how functionally rich a context management technological tool must be and, as a result, how complex it is to design and create one. Nonetheless, the basic aspect of the context management support tool, both technologically and from a structural basis, is how to portray the context in the form of a concept map that is advanced both in terms of a dynamic reconstruction of a digital document (Pedroni, 2006), as well as offer a sophisticated and codified graphics option to enrich the ability to communicate information that is attributive in nature (size and colors of graphic objects, use of the background as a further element in informational support).

The context management environment, inserted within the operational environment of generative learning or knowledge management, must, first of all, offer the context structure in the form of a graphic map, or graph, and implement on this map the set of functions listed above, not because all the functions actually reference the organizational form of knowledge given in the map, but also because to assign the map the role of interface between the user and cognitive environment implies the immediate and on-going transmittal of the ontological structure itself which becomes at the same time the entrance threshold for exploration, the schematic and summary document of the content and relational fabric underpinning it and, finally, symbol itself of the context and 
identifying element. In other words, the interface of the context management environment founded on knowledge mapping takes on its own learning value that goes beyond and is radically different from mere functional support and demands of ergonomics and efficient interaction. (See Figure 1.)

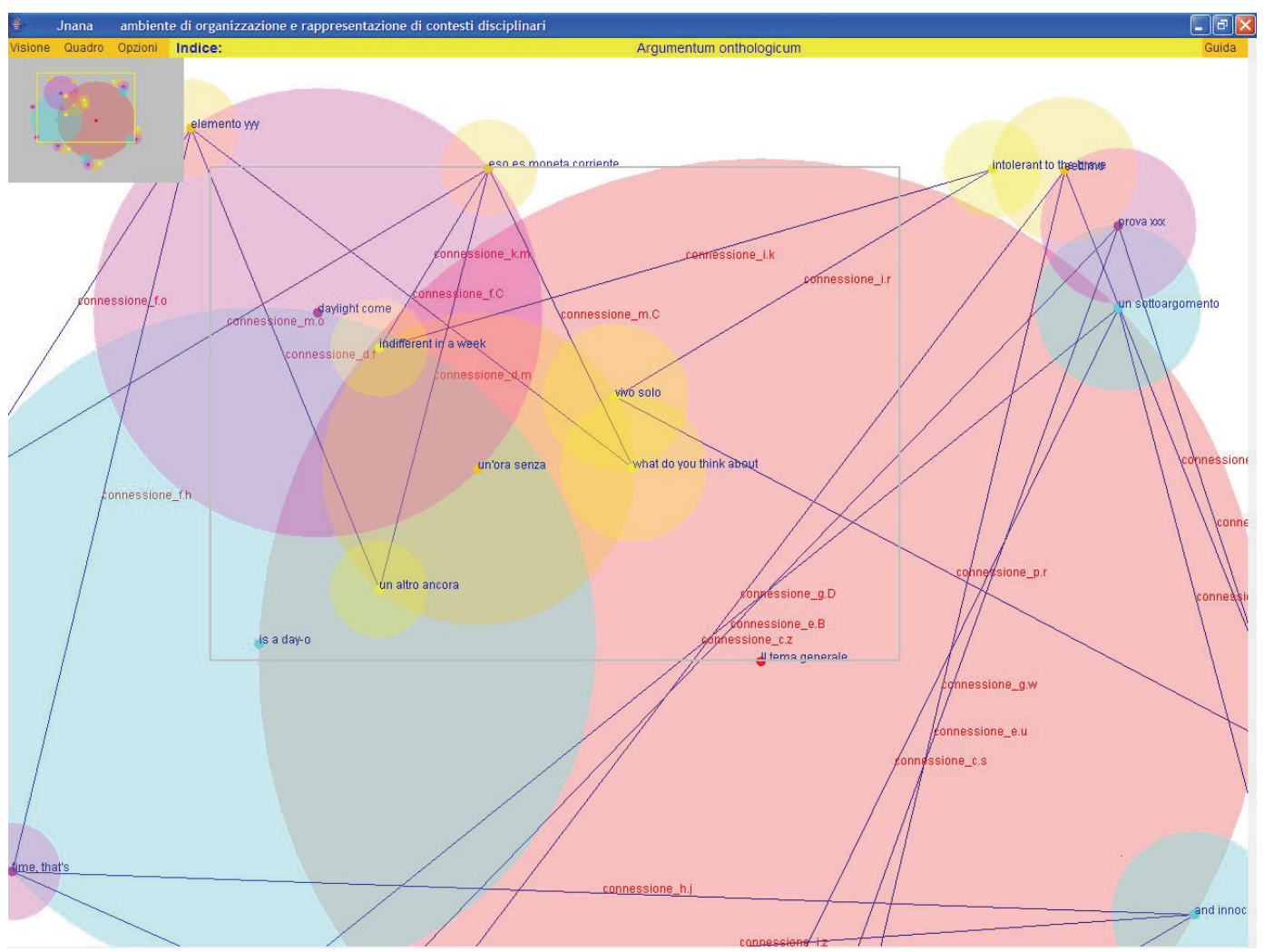

Figure 1: Example of a dynamic, polymorphic and animated conceptual map.

In stressing the articulated aspects of cognitive context mapping, the previous point also implies a sharpening in representational modes. In other words, going beyond the "traditional" limits of the concept map which, in adhering closely to the rules dictated by Joseph Novak and in terms of functional requirements, is made up of software tools based substantially on vector graphics. In its implementation in specific development environments, the concept map is therefore a drawing, or static document, composed of objects (labeled nodes and arcs) sensitive to input events (from CMap Tools to Inspiration, this is the type of document obtained through composition), and, in the best of cases, susceptible to limited graphic modification. The limits of this knowledge mapping mode and the instruments used to implement it, may be summarized as follows:

- the static and rigid nature of the drawing, which is not a limitation in analog documents that have no other options, but becomes one in digital documents which have the established technological need for reconstruction through the combined use of archives or documents structured for storage of data, templates or style sheets to preserve formatting modes, and software tools capable of document parsing and processing by picking up and linking data and formatting. As digital documents, concept maps may be dynamically reconstructed, starting from the use of concept and relation archives (Pedroni, 2004-2006), and because of this potential, may undergo differentiated reconstructive processes for starting conditions or parameterizations that lead to visualization results that are different, although they correspond structurally. A significant example of the versatility of dynamic maps is the regeneration of polymorphic maps belonging to the same cognitive environ- 
ment, starting from the initial position of a different concept (possible, obviously, in graphs in which the reconstruction algorithm is "hierarchical", i.e., in which the first concept examined is placed in a pre-set position and the subsequent development of the structure is derived from the reiteration of the tracking and placement of concepts near those examined) or by random distribution of elements (possible in graphs in which the assigning of a value of attraction between relations makes it possible to apply "gravitational" algorithms that move the elements within the document space until a point of equilibrium is found, in which the attraction between relations is represented in an acceptably correct manner in terms of the distance between the various nodes, i.e., the lengths of their respective arcs);

- the fixed nature of the drawing that advanced interactive tools - both in desk-top applications and, more importantly in this environment, in web-based applications - can modify through forms of animation such 2- or 3-dimensional rotation, zoom and paneling (Pedroni, 2006), but only if the drawing is vectoral and the management of input events allows for this type of function (See Figure 2);

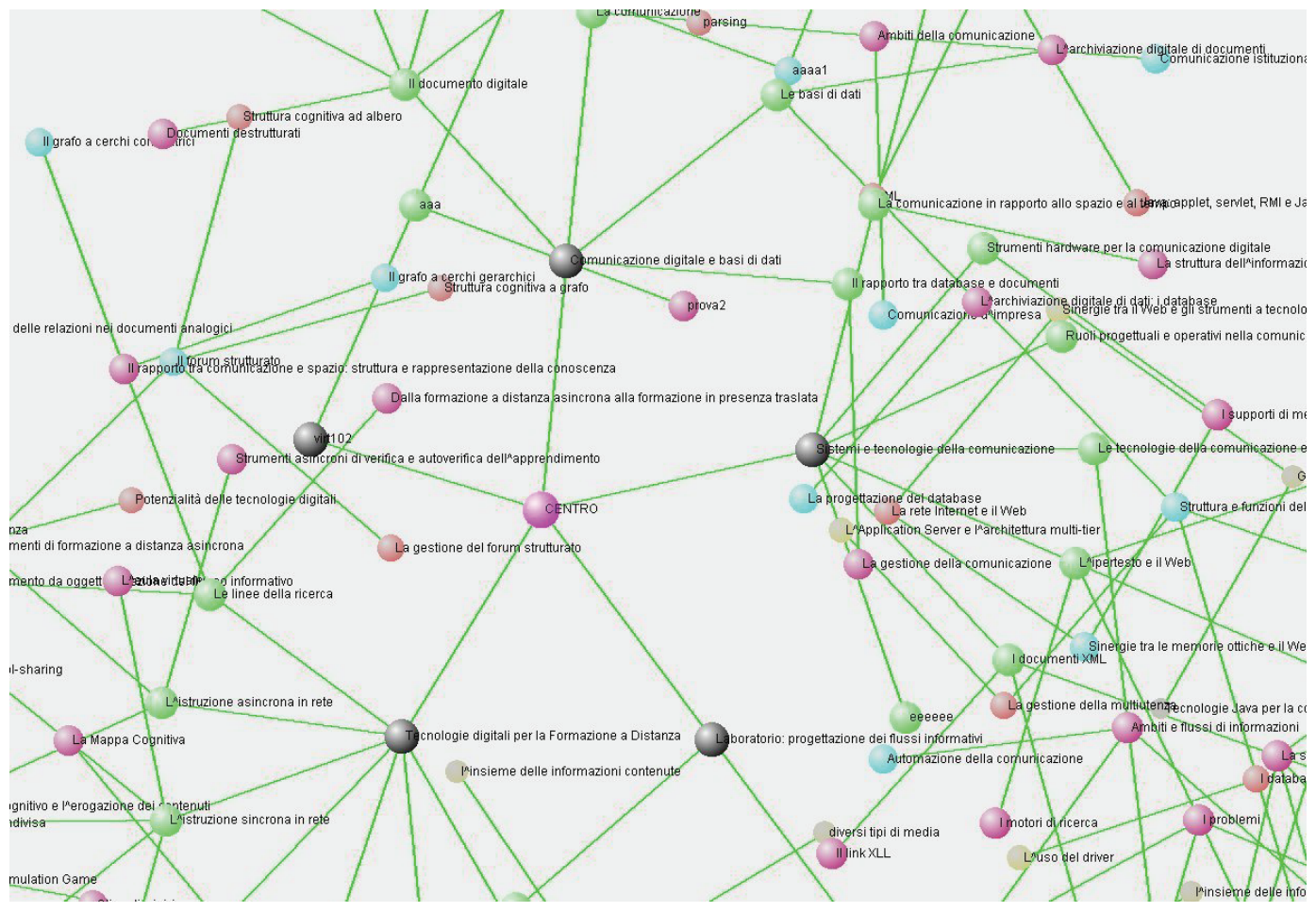

Figure 2: Example of a dynamic, polymorphic, 3D animated conceptual map (without relation labels).

- the differentiation of graphic attributes, in node/concepts (shape, color, size, border color and thickness) arc/relations (color, thickness, line type) and background, whose neutrality from the standpoint of transmitting information may take on significance (through the use of color or special filigreed detailing) in terms of general context-related information, for example, the level of connection internal to the graph (i.e., the relationship between the number of arcs and number of nodes), the structural depth level, the estimated level of difficulty for content exploration (we have excluded from these possible examples all information that may be represented within the map itself, such as afferents to transversal cognitive environments or those external to the one under consideration). 


\section{Interactive Functions}

Once defined the interface tool, which also becomes a content support (in addition to representing its content, the context of a structure also becomes part of it) and guide to exploring topic or corporate knowledge, in addition to the polymorphic dynamic reconstruction and animation of the map itself explained previously, the functions the map must implement are connected to the definition of "context management" already discussed.

The first function is as support for being able to take advantage of content documentation whose archiving is correctly organized by being located within a structural element (similarly to that already permitted by CMap Tools, for example). The significance of contextualizing documental contributions is obvious and is even more effective if accompanied by a short explanatory text, as provided for in Field Trip, a learning object model based on the classification and re-use of web resources in a form organic to the cognitive environment in question. The problem of attributing authorization for content placement is connected to the individual situations in which they are used. In a formal training environment, it may be presumed that this authorization is reserved for the instructor or content expert, or at least that this figure has the role of moderating this authorization process.

Correlated to this function is that of supporting dialogue pertaining to individual topics-concepts represented in the map. Being subject to mapping of the topic context and the need to precisely select in advance the argument the intervention the user will insert, are the basic differences between the normal web-forum and a structured forum environment (Pedroni, 2006), a tool which, from a functional standpoint, diverges from constructivism to converge with a rationalization of the aggregation of the most significant knowledge within the context of knowledge management (structuring of interventions in relation to their placement in a pre-ordered grid is aimed at avoiding repetition of questions and answers so frequent in normal web-forums and which certainly does not represent an incentive for dialogue aimed at the collaborative construction of knowledge characteristic of constructivism and in which communicational redundancy is an integral feature and positively viewed). In addition, it is a good idea to avoid an ambiguous identification of the context management environment through the simple and limited functionality of a structured forum (Pedroni, 2006) that overshadows the constructivist nature tied to dynamic re-composition and permanent structural modifiability of the graph. If, in fact, the dialogue interaction, in aiming for synthesis, efficiency and avoidance of redundancy, becomes removed from constructivist paradigms, context management fully grasps its significance and potential through common construction of the structure of concepts and relations and in the inherent negotiation of meanings.

This is the third function specific to the context management technological support environment. Naturally, on the basis of a specific policy for handling authorizations, the structure composition may be the object of modifications, or proposals for interactive modification, i.e., the same initial functions of composition of the map can remain and allow for its continuous revision. The preservation of a primary version, or one officially recognized by the expert responsible for the subject-related content or organizational knowledge, and the registration (moderated or unmoderated) of the proposals for structural alteration or analysis, can reach an advanced level of refinement through the composition and use of a second network parallel to knowledge mapping and designed to support the stake-holders in the reconstructive process and their relations. The connections between the two networks, links whose significance lies in the testimony of the proposals of those dialoguing, may be of tangible help in the progressive analysis of cognitive environments (Pedroni, 2006). 


\section{Integrability}

Both in the case of management of the subject-related ontological structure (its application in a strictly learning environment), as well as the organization of the system of presenting and socializing knowledge (its application in institutional or corporate operational environments), context management must necessarily answer the demands for usability, scalability and, most importantly, the ability to integrate itself within pre-existing methodological and technological structures (often deeply entrenched) that characterize the environments in which it is implemented. These exigencies may be summarized in the following five points:

- ergonomy: the interface based on the full-screen mapping of the context must be intuitive, primarily in the use of enlargement, zoom, paneling, animated rotation and polymorphic dynamic reconstruction, functions that are indispensable in frequent cases of highly-complex graphs, and complete from the standpoint of functional support without being invasive, i.e., without attracting attention away from the map itself;

- efficacy: there must be many modes of knowledge mapping in order to effectively meet the needs of context comprehension and interpretation, and they must also be able to be used in various ways in order to facilitate user exploration and interactivity. Among the reference graphic models tested as part of the CARID project at the University of Ferrara, special note should be made of the concentric circle, hierarchical circle and rotational 3-D cone, all noteworthy for clarity of design, ability to handle significant numbers of elements and a high level of internal links, their reconstructive dynamism and wealth of options for display and animation and, more generally, for their overall appearance. All of these models are derived from hierarchical reconstructive algorithms and the 3-D graph is derived from gravitational algorithms;

- efficiency: the usability of this tool is based in real contexts involving integrability with other pre-existing technological environments, in particular with databases and structured document repository systems. As a result, their efficiency must be optimized from this standpoint by implementing, on one hand, a system for storing information regarding map reconstruction not based on the use of the internal database, but on XML, RDF and OWL protocols (respectively, eXtensible Markup Language, Resource Definition Framework and Ontology Web Language, structured document definition protocols) on the basis of information supplied by the W3C consortium. On the other hand, by implementing the libraries and tools for updating to data exchange standards with other software resources available online, in particular the Web Services standard that includes the SOAP (Simple Object Access Protocol) and WSDL (Web Services Description Language) protocols, and other learning object tracking standards used in learning environments, in particular SCORM (Sharable Content Object Reference Model);

- completeness: the structural level of the map (improvement and analysis of all the concepts that comprise it and the relational fabric that connects them) must not only correctly and completely represent the cognitive unit in question so that it may be interpreted easily by users, but also to fully organize and arrange all documental and informational resources regarding the context internal to the system itself and available online, in order to optimize research activities and its fruition;

- flexibility: through conformity with standard protocols and internal features of the software, all the above requisites must adapt to the characteristics of the pre-existing information systems in the environments in which the context management is implemented. In other words, the integrability of the context management system with the corporate data warehouse and active procedures, or with the learning interaction environment, is the 
primary condition for concretely promoting its use and diffusion. Integrability derives from the flexibility with which context management conforms to the modalities and formats of documental and information archiving and fruition through configuration procedures which must unite the characteristics of simplicity and immediacy of use with functional integration performance.

\section{Conclusions}

The development of context management tools which CARID at the University of Ferrara has been researching for years, in an evolutional axis of e-learning and knowledge management that is important but often misinterpreted or misunderstood, primarily for the cultural difficulty encountered in spreading structural models for knowledge reconstruction and representation that go beyond sequence and hierarchy to place at the center of attention not the set of concepts but the relational fabric, the true element that identifies and characterizes the structure. On the other hand, if one intends respecting the complexity and not give in to the temptation of simplification that ends up causing perceptible loss of information, negotiation of meaning and metacognition during analysis of a knowledge context must necessarily include use of tools that make it possible to manage (and therefore to collaboratively construct, organize represent, transmit) the conceptual-relational context.

The first tools, from which previous images were taken, have led to a number of errors in design and fruition strategy, giving rise to previous comments regarding context representation, interaction management and integrability. During 2007, it is expected that a prototype for context management of the environment will be completed that will act as the fourth sustaining axis in learning environments, with the function of transmitting content, learning interaction and process control, and be a basic tool for knowledge management systems in the institutional and corporate environment.

\section{References}

Canas,A. J., Hill, G., Carff, R., Suri, N., Lott, J., Eskridge, T., Gomez, G., Arroyo, M. \& Carvajal, R. (2004). CmapTools: A knowledge modeling and sharing environment. 1st International Conference on Concept Mapping, Pamplona: Direccion de publicaciones de la Universidad Publica de Navarra

Canas, A. J., Hill, G., \& Lott, J. (2003). Support for constructing knowledge models in CmapTools (Technical Report IHMC CmapTools2003-02). Pensacola, Florida: Institute of Humanand Machine Cognition.

Fourie, L., Schilawa, J., \& Cloete, E. (2004). The value of concept maps for knowledge management in the banking and insurance industry: A German case study. 1st InternationalConference on Concept Mapping, Pamplona: Direccion de publicaciones de la Universidad Publica de Navarra

Gonzales, G., \& Artiles, S. (2004). Simetria de la tecnica de mapas conceptuales y la dimension informacional de la gestion de conocimiento de la s organisaciones: GECYT como caso de studio. 1st InternationalConference on Concept Mapping, Pamplona: Direccion de publicaciones de la Universidad Publica de Navarra

Kremer, R. (1994). Concept mapping: Informal to formal. In W. Tepfenhart, J. Dick, \& J. Sowa (Eds.), Proceedings of the Third International Conference on Conceptual Structures, Knowledge Acquisition Using Conceptual Graphs Theory Workshop. University of Maryland College Park, MD., Aug 19, 1994. pp. 152-167.

Novak, J. D. (1998). Learning, creating, and using knowledge: Concept Mapsas facilitative tools in schools and corporations. Mahweh, NJ:Lawrence Erlbaum Associates.

Novak, J. D., \& Gowin, D. B. (1984). Learning how to learn. New York:Cambridge University Press. 
Pedroni, M. (2004). Dalla mappa concettuale alla scrittura a grafo. In Atti Didamatica 2004, Ferrara: Omniacom

Pedroni,M. (2005). Knowledge mapping attraverso la ricostruzione automaticadi mappe concettuali dinamiche. 10th Knowledge Management Forum. Siena, Italy. Barabasi, A.L. (2004). La scienza delle reti, Torino: Einaudi

Pedroni, M. (2006). e-learning e rappresentazione della conoscenza, Ferrara: Tecomproject

Tamayo, M. F. A. (2004). El mapa conceptual: Un texto a interpretar. 1st International Conference on Concept Mapping, Pamplona: Direccion de publicaciones de la Universidad Publica de Navarra

Tillman, W., \& Wissmann, J. (2004). Dynamic concept maps for music. 1st International Conference on Concept Mapping, Pamplona: Direccion de publicaciones de la Universidad Publica de Navarra

Trentin, G. (2004). Apprendimento in rete e condivisione delle conoscenze, Milano: Franco Angeli

\section{Biography}

Marco Pedroni is the head of the computer systems unit of CARID (Centro di Ateneo per la Ricerca, l'Innovazione Didattica e l'Istruzione a Distanza dell'University degli Studi di Ferrara University Center for Research, Learning Innovation and Distance Learning at the University of Ferrara) and professor of undergraduate, masters and specialist/training programs run by CARID in e-learning methods. His specific areas of competence and research are knowledge management, online structures and their representation using concept maps, and information and document flows. 